\title{
Diagnosis, management, and pathophysiology of post-Fontan hypoxaemia secondary to Glenn shunt related pulmonary arteriovenous malformation
}

\author{
R Premsekar, J L Monro, A P Salmon
}

\begin{abstract}
An 8 year old child with tricuspid atresia had developed right sided pulmonary arteriovenous malformations following a previous classic Glenn procedure. These became clinically manifest immediately after Fontan conversion because of severe systemic desaturation. The pathophysiology and postoperative medical management of this case is described and related to current understanding of the aetiology of acquired pulmonary arteriovenous malformations following cavopulmonary shunt.

(Heart 1999;82:528-530)
\end{abstract}

Keywords: classic Glenn shunt; bidirectional cavopulmonary anastomosis; pulmonary arteriovenous malformations; Fontan circulation

Superior cavopulmonary anastomosis was first described by Carlon in $1951^{1}$ and has been in use since the first clinical application by Glenn and Patino in $1954 .^{2}$ Although the initial results were impressive, most patients became increasingly cyanosed during medium and long term follow up. Macroscopic pulmonary arteriovenous malformations (PAVMs) accounted for this in a minority of cases but progressive desaturation developed in the absence of angiographically proven fistulae. ${ }^{3}$ The original operation involved end to end anastomosis of the disconnected right pulmonary artery to the transected superior vena cava, but since the 1980s the superior vena cava has been attached to the right pulmonary artery with preservation of pulmonary artery continuity (bidirectional cavopulmonary anastomosis (BCPA)). Recent evidence suggests intrapulmonary shunting is inevitable after $\mathrm{BCPA}^{4}$ although the degree may be dependent on the presence and amount of competitive flow. It has now been shown that macroscopic PAVMs are merely the end stage of a gradual process of increasing perfusion mismatch associated with loss of hepatic factor. $^{4-6}$ We report a patient with a classic Glenn shunt in whom the abnormal arteriovenous shunting in the right lung led to severe desaturation following the Fontan procedure.

\section{Case report}

The patient underwent pulmonary artery banding for tricuspid atresia in the neonatal period. The band subsequently distorted the right pulmonary artery and there was an increased resistance in the left lung. In order to protect the right pulmonary artery, a classic Glenn shunt was undertaken and the band to the left pulmonary artery tightened at 2 years of age. Haemodynamics at 7 years of age were: systemic saturation $75 \%$, right pulmonary vein $76 \%$ (confirming ventilation-perfusion mismatch in the right lung), and left pulmonary vein $99 \%$. Mean pressure in the superior vena cava and right pulmonary artery was $11 \mathrm{~mm} \mathrm{Hg}$, and $19 \mathrm{~mm} \mathrm{Hg}$ in the left pulmonary artery.

The patient underwent a lateral tunnel Fontan with fenestration together with restoration of pulmonary artery continuity at 8 years of age. Coming off bypass the systemic saturation fell to $40 \%$. The fenestration was presumed to be too large and after re-establishing bypass it was closed. The saturation remained critically reduced (30-40\%) and we speculated that this was related to preferential flow to the right lung (low resistance and ventilation-perfusion mismatch) and reduced flow to the left (higher resistance but normal ventilation-perfusion). In theatre, an intravenous prostacyclin infusion and inhaled nitric oxide improved oxygenation to $60 \%$ and the patient was transferred to the intensive care unit. $\mathrm{He}$ was weaned off the prostacyclin over two days but remained extremely sensitive to the nitric oxide. During this period the central venous pressure was only $6 \mathrm{~mm} \mathrm{Hg}$.

Cardiac catheterisation four days after surgery confirmed the disordered physiology and a good surgical repair. There was no evidence of pulmonary artery distortion, the lateral tunnel anastomosis was satisfactory, and there was no residual intracardiac shunting. Lateral tunnel injection showed heavy preferential flow to the right lung with minimal flow to the left pulmonary artery (fig 1), and we were initially concerned that there may be a severe stenosis of the left pulmonary artery origin. There was evidence of a diffuse perfusion abnormality causing a generalised "fluffy" appearance with macroscopic fistulae predominantly in the right
Dr Salmon.

Accepted for publication 27 April 1999 
lower lobe (figs 1 and 2). Furthermore the right pulmonary veins filled almost immediately ( $<1.5$ cardiac cycles), confirming notable intrapulmonary shunting. Contrast injection at the junction of the lateral tunnel with the pulmonary arteries excluded a stenosis of the left pulmonary artery which was widely patent, and there was a normal vascular appearance on that side (fig 2). Selective left pulmonary artery angiography also confirmed normal pulmonary perfusion with filling of the left pulmonary veins occurring only after six cardiac cycles.

The patient was extubated two days postoperatively into headbox nitric oxide which he required for a total of 10 days until his saturations were $75 \%$ in air; he was discharged home on the 18 th postoperative day. Repeat catheter study at one month showed systemic saturations of $90 \%$ in air, a central venous pressure of $12 \mathrm{~mm} \mathrm{Hg}$, and complete resolution of the perfusion abnormality in the right lung. There was symmetrical perfusion of both lungs from

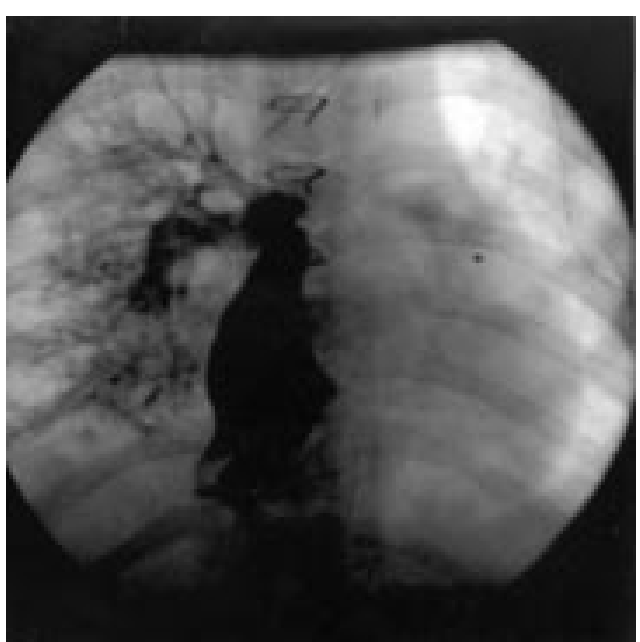

Figure 1 Contrast injection in the lateral tunnel showing preferential flow to the right lung which demonstrates a grossly abnormal vascular pattern and almost no flow to the left lung.

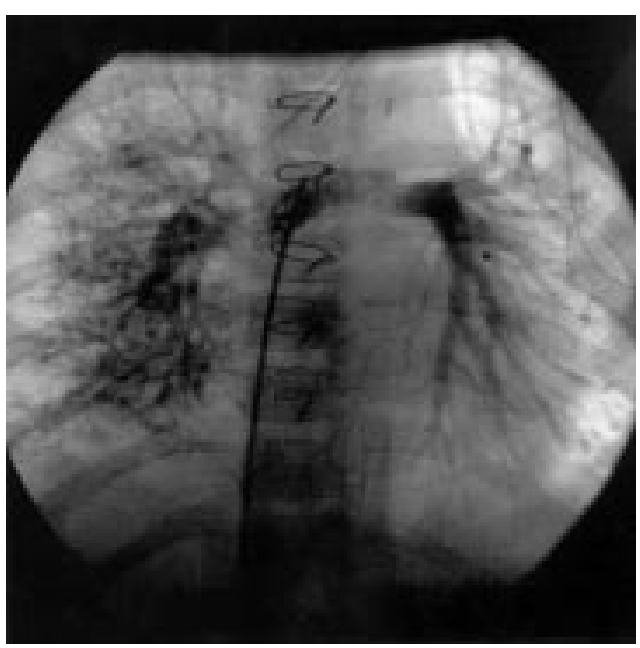

Figure 2 Angiography five days post-Fontan connection. Injection at the junction of the lateral tunnel with the branch pulmonary arteries confirms patency of left pulmonary artery with a normal pulmonary vascular pattern. The abnormal vascular pattern on the right is again evident.

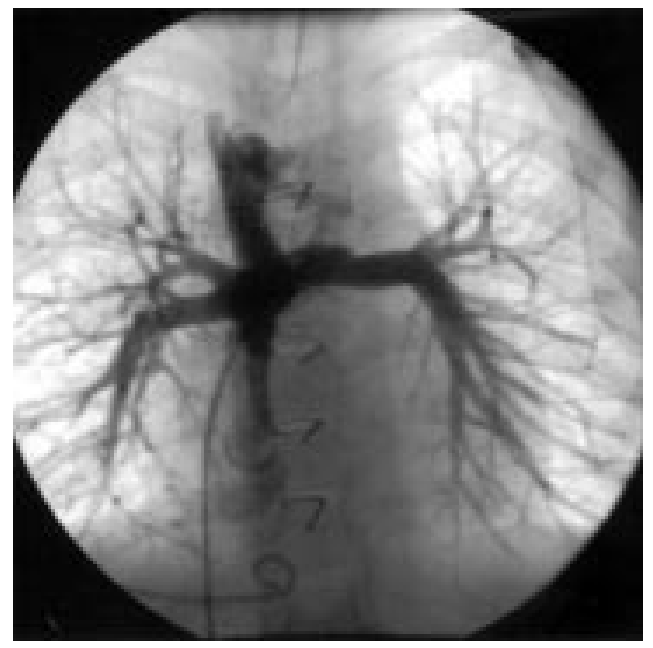

Figure 3 Repeat angiography four weeks post-Fontan connection with complete resolution of the arteriovenous malformations in the right lung and symmetrical perfusion of both lungs.

the lateral tunnel and no evidence of early filling of the right or left pulmonary veins (fig 3).

\section{Discussion}

Pulmonary arteriovenous fistulae are a well established complication following both the classic Glenn shunt ${ }^{3}$ and the BCPA. ${ }^{4}$ Macroscopic PAVMs are known to occur most commonly in the right lower lobe and this was previously felt to be related to the non-pulsatile blood flow and its inability to counteract the effects of gravity. ${ }^{7}$ Diminished flow to the upper lobe was also thought to be a result of hypoxaemia and pulmonary vasoconstriction. ${ }^{7}$ However, it has now been recognised that exclusion of normal hepatic venous return from the pulmonary arterial circulation is associated with the formation of PAVMs, ${ }^{4-6}$ which may or may not become macroscopic. ${ }^{3}$ We believe that the predominance of macroscopic PAVMs in the lower lobe, exemplified in our patient (fig 2), is not related to mechanical factors alone but their superimposition on a generalised pulmonary abnormality ("microscopic" PAVMs) caused by abnormal vasodilatation. The association of vasodilatation with PAVMs is confirmed in our patient. There was heavy preferential flow to the affected lung and the central venous pressure was exceptionally low in the immediate postoperative period ( $6 \mathrm{~mm} \mathrm{Hg}$ ). Furthermore, coincident with clinical and angiographic resolution of the PAVMs, the central venous pressure increased to a more normal Fontan level $(12 \mathrm{~mm} \mathrm{Hg}$ ), suggesting progressive pulmonary vasoconstriction. Functional dilatation is presumably a result of the endogenous production of a vasodilatory substance, possibly nitric oxide $^{8}$ or prostaglandins. ${ }^{9}$ Changes in the splanchnic vascular perfusion associated with portal hypertension in cirrhosis have been shown to be at least partially caused by excess formation of nitric oxide. ${ }^{8}$ Krowka and colleagues suggest that patients with liver dysfunction may be unable to metabolise or clear a pulmonary vasodilator that originates in the mesenteric circulation. ${ }^{10}$ 
Although we were aware of abnormal arteriovenous shunting in the right lung preoperatively, the patient had adequate systemic saturations $(75 \%)$ because there was more than adequate blood flow to the unaffected left lung. After surgery there was preferential flow to the right lung and this "steal" effect may have been exacerbated by a slightly higher than normal resistance in the left lung. We managed the cyanosis by attempting to reduce the resistance and increase flow to the left lung to improve the overall ventilation-perfusion characteristics. The nitric oxide appeared to have the most beneficial effect.

Embolisation of the PAVMs has been reported to correct ventilation-perfusion mismatch in the affected lung, ${ }^{11}$ but we believed that reintroduction of hepatic venous flow to the right lung would result in spontaneous resolution of the PAVMs. Firstly, PAVMs in patients with chronic liver disease and cyanosis (hepatopulmonary syndrome) show reversal of ventilation perfusion abnormalities after liver transplantation. ${ }^{10}$ Secondly, orthotopic heart transplantation has resulted in prompt resolution of pulmonary arteriovenous malformations in a child who had previously undergone a Kawashima operation, where hepatic venous return had been excluded from the pulmonary circulation. ${ }^{12}$ Lee and colleagues describe a case of a 6 year old child with anomalous hepatic venous drainage to the left atrium and an intact atrial septum developing PAVMs which resolve spontaneously after redirection of hepatic drainage to the right atrium.

Few patients now undergo the classic Glenn shunt as the BCPA is established as providing excellent palliation before Fontan completion. However, it is known that PAVMs also occur following BCPA and it presumably occurs bilaterally in those patients where the superior vena cava is the only contributor to blood flow to both lungs. Following a Fontan in a patient with a previous BCPA and PAVMs, cyanosis may be a problem but should be less so than preoperatively because of increased pulmonary blood flow. We would strongly recommend supportive medical management in the postoperative period to allow time for spontaneous resolution of PAVMs.

1 Carlon CA, Mondini PG, De Marchi R. Surgical treatment of some cardiovascular diseases (a new vascular anastomosis). F Int Coll Surg 1951;15:1-11.

2 Glenn WWL, Patino JF. Circulatory by-pass of right heart. Preliminary observations on direct delivery of venacaval Preliminary observations on direct delivery of venacaval
blood in to pulmonary artery shunt. Yale f Biol Med 1954; blood in to

3 Mathur M, Glenn WWL. Long term evaluation of cavopulmonary artery anastomosis. Surgery 1973;74:899-916.

4 Bernstein HS, Brook MM, Silverman NH, Bristow J. Development of pulmonary arteriovenous fistulae in children after cavopulmonary shunt. Circulation 1992;92(suppl II):309-14.

5 Lee J, Menkis AH, Rosenberg HC. Reversal of pulmonary arteriovenous malformation after diversion of anamolous hepatic drainage. Ann Thorac Surg 1998;65:848-9.

6 Srivastava D, Preniinger T, Lock JE, et al. Hepatic venous blood and the development of pulmonary arteriovenous malformations in congenital heart disease. Circulation 1995;92:1217-22.

7 Boruchov IB, Swenson EW, Elliot LP, et al. Study of the mechanism of shunt failure after superior vena cava-right pulmonary artery anastomosis. I Thorac Cardiovasc Surg 1970;60:531-9.

8 Pizcueta P, Pique JM, Fernandez M, et al. Modulation of the hyperdynamic circulation of cirrhotic rats by nitric oxide inhibition. Gastroenterology 1992;103:1909-15.

9 Shijo H, Sasaki H, Yuh K, et al. Effects of indomethacin on hepatogenic pulmonary angiodysplasia. Chest 1991;99: 1027-9.

10 Krowka MJ, Cortesa DA. Hepatopulmonary syndrome: an evolving prospective in the era of liver transplantation. Hepatology 1990;11:138-42.

11 Gomes A, Benson L, George B, et al. Management of pulmonary arteriovenous fistulas after superior vena cava-right pulmonary artery (Glenn) anastomosis. $\mathcal{f}$ Thorac Cardiovasc Surg 1984;87:636-9.

12 Garham K, Sondheimer H, Schaffer M. Resolution of cavopulmonary shunt-associated pulmonary arteriovenous malformation after heart transplantation. 7 Heart Lung Transplant 1997;16:1271-4. 